\title{
The perceived structure of social attitudes*
}

\author{
CARNOT E. NELSON† \\ University of South Florida, Tampa, Florida 36620
}

\begin{abstract}
The purpose of this study was to determine the multidimensional structure of perceived social attitudes. Forty-five Ss described approximately 10 different persons they knew by selected attitude statements from a list supplied by E. A measure of statement concurrence for each pair of statements was derived from these data and was used in Kruskal's multidimensional scaling program. The fit for the 3-dimensional configuration fell in the "fair" range with additional dimensions adding little to the goodness of fit. This configuration was interpreted by finding axes in the configuration which corresponded to independently measured psychological properties of the statements. These axes were located by multiple-regression techniques. Liberal-conservative and moderate-extreme provided a satisfactory description of two of the three dimensions. The third dimension was uninterpretable.
\end{abstract}

Person perception involves the perception by one individual of many facets of another individual from his emotions to his personality, attitudes, or beliefs. An important characteristic of these perceptions is that they are structured (Hastorf, Schneider, \& Polefka, 1970). A number of investigators have been concerned with the perceived structure of emotions (e.g., Ableson \& Sermat, 1962; Frijda, 1969; Schlosberg, 1952, 1954; Woodworth, 1938) and personality trait adjectives (e.g., Jackson, 1962; Jackson, Messick, \& Solley, 1957; Rosenberg, Nelson, \& Vivekananthan, 1968; Rosenberg $\&$ Sedlack, 1972). Both lines of reseach have shown that these two aspects of others have stable structures which can be described using multidimensional scaling.

Messick (1956) was the first investigator to use multidimensional scaling techniques to study the perceived structure of social attitudes. Using attitudes toward war, capital punishment, and treatment of criminals, he found that (1) the perceived relationships could be adequately represented in dimensional terms, and (2) the configuration of seminary students and Air Force officer candidates were almost identical. Boyd and Jackson (1967) investigated attitudes designed to reflect three hypothesized dimensions of authoritarian ideology. Using interstimulus similarity judgments and multidimensional scaling, they found that subsets of attitude statements reflecting the hypothesized facets were perceived as representing independent dimensions.

These studies dealing with the perceived structure of social attitudes have shown that the structure of social attitudes can be viewed in multidimensional terms. However, each of the studies has involved only a few attitudes. Thus, we have no general description of the perceived structure of social attitudes but rather a description of small portions of the structure.

Some notion as to the possible structure of perceived attitudes can be gained from examining studies

*This research was supported by a grant from the University of South Florida and is sponsored by Dewey Rundus who takes full editorial responsibility for its contents.

tR equest for reprints should be sent to Carnot $E$. Nelson, Department of Psychology, University of South Florida, Tampa, Florida 33620 . concerning the actual structures of these attitudes. Kerlinger (1967) claimed that the structure of social attitudes is dualistic. That is, there are two basic orthogonal dimensions which span the attitude domain but there is little bipolarity. One of the dimensions is labeled liberal and the other conservative. Thus liberal-conservative are not bipolar ends of the same dimension but rather are two orthogonal dimensions. Hicks and Wright (1970) found liberal-conservative to be a complex dimension comprised of at least four independent liberal-conservative dimensions: Economic, political, religious, and esthetic. Therefore, it seemed probable that liberal-conservative would account for at least one of the dimensions. However, it was unclear as to how many other dimensions would be needed to describe the structure and the nature of these dimensions. The present study, therefore, had as its primary objective discovering the dimensionality and dimensions of the perceived structure of social attitudes.

\section{METHOD}

The method and analyses used in the present study were similar to that used by Rosenberg, Nelson, and Vivekanathan (1968) in studying the perceived structure of personality trait adjectives.

Forty-five students in introductory psychology were asked to describe 10 persons they knew (e.g., friends, relatives, public figures) in terms of 100 attitudes statements, by placing in one category all the statements with which a particular individual would agree. The attitude statements consisted of 10 items from 10 different Thurstone-type scales. The scales dealt with attitudes toward the following objects: Reality of God, divorce, birth control, patriotism, law, sex information, war, punishment of criminals, censorship, and mental hospitals. Ss distributed the statements among the people in any way they desired, except that each statement could be used in only 1 description. In addition, Ss were provided with a miscellaneous category for statements "which did not go with any other."

The sorting data were used to calculate a measure of the degree of psychological relatedness between each of the statements. To measure the degree of psychological relatendess the disassociation measure developed by Rosenberg et al (1968) was used. As a preliminary step, a "disagreement score" was obtained for each pair of traits by counting the number of Ss who ascribed the two statements to two different persons they 
had in mind. For example, if 5 of 45 Ss assigned the same two statements to the same person, the "disagreement score" for this pair of statements would be 40 . Any statement assigned to the miscellaneous category was considered as being in its own catetory. The disassociation measure," $\delta \mathrm{ij}$, was then computed from $S_{\mathrm{ij}}$ namely:

$$
\delta_{\mathrm{ij}}=\sum_{\mathrm{k}=1}^{\mathrm{T}}\left(\mathrm{S}_{\mathrm{ik}}-\mathrm{S}_{\mathrm{jk}}\right)^{2}
$$

where $T$ is the number of attitude statements and $\mathrm{K}$ is an index for each of the statements. The disassociation measures were used as input into Kruskal's $(1964 \mathrm{a} ; 1964 \mathrm{~b})$ nonmetric multidimensional scaling program, which then produced a configuration of statement points in a multidimensional space.

In order to interpret the multidimensional solutions, 160 different Ss rated each of the statements along one of four 7-point scales. These scales were: liberal-conservative, social liberal/social conservative, political liberal/political conservative, and moderate-extreme. The median ratings for each statement was used in a multiple regression analysis to relate each of the four properties to the multidimensional solutions.

\section{RESULTS AND DISCUSSION}

The dimensionality of the space was evaluated by examining the stress values, Kruskal's goodness-of-fit index which reflects how well the data map into a space of any given dimensionality. The stress values in the present study for the one-, two-, three-, and four-dimensional solutions were $42 \%, 22 \%, 14 \%$, and $10 \%$, respectively. Because there was little decrease in stress after three dimensions and the stress in two dimensions was high, attempts at interpretation were limited to the three-dimension solution. Although the stress values obtained here were slightly higher than those found in the studies of personality traits (Rosenberg et al, 1968; Wing \& Nelson, 1972), they were substantially below that of random input (Stenson \& Knoll, 1969), indicating that the data can be multidimensionally structured.

Linear multiple regression was used to locate axis in the three-dimensional attitude statement space which corresponded to the rated properties of the statements. For each property, the linear method locates an axis such that the linear correlation between the external ratings and the values of the statements is maximized. The linear method is based on linear multiple regression so that an ordinary multiple $\mathrm{R}$ provides a quantitative estimate of the degree to which a property actually corresponds to a dimension in the trait space.

The multiple $\mathrm{R}$ for each of the properties and its best fitting axis were: Liberal-conservative .966, social liberal-conservative .958 , political liberal-conservative .964 , and moderate-extreme .800 . There are obviously no absolute standards for deciding when an $R$ is sufficiently large so that an external property is acceptable as an interpretation of a dimension. The standard generally used for validity coefficients, that is a correlation of at least .7, seems appropriate here also.
Using the value of .7 as a minimum, all of the properties can be used to interpret the solution.

In order to interpret the solution, the various properties should be orthogonal to one another. The three liberal-conservative dimensions were not orthogonal as the angles between them ranged from 11 to $12 \mathrm{deg}$ indicating that, in essence, they were describing the same dimension. However, the angle between the three liberal-conservative and the moderate-extreme dimensions ranged from 83 to $86 \mathrm{deg}$, indicating that these were separate dimensions. Thus, two of the dimensions in the three-dimensional solution were liberal-conservative and moderate-extreme.

The third dimension proved uninterpretable. This is not unusual as Rosenberg et al (1968) could not specify in their study of personality traits a third property in addition to social and intellectual desirability.

The lack of difference between the social and political liberal-conservative dimensions and the uninterpretability of the third dimension may be due to two methodological problems. First, the sample of attitude statements selected may not have adequately represented the full domain of attitudes. Second, although Wing and Nelson (1972) showed that using a trait more than once did not change the results in the area of personality traits, the technique of allowing a $S$ to use each statement in only one description may have forced him into making stereotypic descriptions. Such descriptions may have forced the Ss into grouping all types of liberals or conservatives into one category.

\section{REFERENCES}

Abelson, R. P., \& Sermat, V. Multidimensional scaling of facial expressions. Journal of Experimental Psychology, 1962, 63, 546-554.

Boyd, J. D., \& Jackson, D. N. The perceived structure of social attitudes and personality: A multidimensional approach Multivariate Behavioral R esearch, 1967, 2, 280-297.

Frijda, N. H. Recognition of emotion. In L. Berkowitz (Ed.), Advances in experimental social psychology. Vol. 4, New York: Academic Press, 1969.

Hastorf, A. H., Schneider, D. J., \& Polefka, J. Person-perception. Reading, Mass: Addison-Wesley, 1970.

Hicks, J. M., \& Wright, J. H. Convergent-discriminant validation and factor analysis of five scales of liberalism-conservatism. Journal of Personality \& Social Psychology, 1970, 14 114-120.

Jackson, D. N. The measurement of perceived trait relationships. In D. Miller (Ed.), Decisions, values and groups. Vol.2 New York: Pergamon Press, 1962.

Jackson, D. N., Messick, S., \& Solley, C. M. A multidimensional scaling approach to the perception of personality. Journal of Psychology, 1957, 44, 311-318.

Kerlinger, F. N. Social attitudes and their critical referents: A structural theory. Psychological Review, 1967, 74, 114-120.

Kruskal, J. B. Nonmetric multidimensional scaling: I Psychometrika, 1964, 29, 1-27(a).

Kruskal, J. B. Nonmetric multidimensional scaling: II Psychometrika, 1964, 29, 115-129(b).

Messick, S. J. The perception of social attitudes. Journal of Abnormal \& Social Psychology, 1956, 52, 57-66.

Rosenberg, S., Nelson C. \& Vivekananthan, P. S. A multidimensional approach to the structure of personality impressions. Journal of Personality and Social Psychology, 1968, 9, 283-294.

Rosenberg, S., \& Sedlak, A. Structural respresentations of implicit personality theory. In L. Berkowitz (Ed.), Advances 
in experimental social psychology. Vol. 6, New York: Acad emic Press, 1972.

Schlosberg, H. Three dimensions of emotion. Psychological Review, 1954, 61, 81-88.

Wing, H., \& Nelson, C. E. The perception of personality through trait sorting: A comparison of trait sampling techniques. Multivariate Behaviral Research, 1972, 7, 269-274.

Woodworth, R. S. Experimental psychology. New York: Holt, 1938.
NOTE

1. In the study of personality traits no difference was found between using each trait in only one description and using a trait in as many descriptions as the $S$ desired (Wing \& Nelson, 1972).

\title{
Effects of thirst drive on cue utilization and cue dominance of spatially separate cues in albino rats*
}

\author{
JEROME S. COHEN and GISELLE TUBARO \\ University of Windsor, Windsor, Ontario, Canada N9B 3P4
}

\begin{abstract}
Albino rats under moderate $(18 \mathrm{~h} /$ day $)$ or high $(23.5 \mathrm{~h} /$ day $)$ water deprivation we re required to learn either a central door cue or peripheral wall cue simultaneous discrimination in a two-choice discrimination apparatus. Following this acquisition phase, a series of redundant-relevant cue trials with both door and wall cues were introduced. Animals were tested on the utilization of wall and door cues separately and on the dominance for each cue. Drive level only affected original discrimination acquisition of the peripheral wall-cue task. Moderate-drive animals were better able to learn the wall-cue discrimination than high-drive animals. In general, door-cue learners were able to utilize both cues but showed dominance for door over wall cues. Wall-cue learners, however, were only able to utilize wall cues and showed dominance for wall over door cues.
\end{abstract}

Drive level has been shown to be inversely related to exploratory activity of rats in reinforced complex mazes (Chapman \& Levy, 1957; Cohen \& Stettner, 1968; Hughes, 1965) and to be inversely related to cue utilization of redundant-relevant cues in discrimination tasks (Bruner, Matter, \& Papanek, 1955; Cohen, Stettner, \& Michael, 1968; Telegdy \& Cohen, 1971). In view of the similar relationships between drive and these two separate behaviors, cue utilization could be considered to be mediated by exploratory activity. Increased exploratory activity under decreased drive level may increase the likelihood that animals would sample more redundant-relevant stimuli. This possibility is suggested by recent findings (Cohen \& Sullivan, 1973) in which only moderately water deprived $(18 \mathrm{~h})$ rats were disrupted in their responses to learned central brightness cues by changes in location of redundant-relevant peripheral cues. The present study is an extension of the recent Cohen and Sullivan research (1973). As such, it represents an indirect test of the proposed exploration mediation hypothesis of cue utilization. Animals under high- $(23.5 \mathrm{~h})$ and moderate-water $(18 \mathrm{~h})$ deprivation were required to learn a brightness discrimination from either central door or peripheral wall brightness cues.

*The present study was conducted under a National Research Council of Canada Grant (APA 7450).
Redundant-relevant peripheral or central cues were then added to the learning environment. Animals were then tested for their utilization of each cue separately and for their cue preference, i.e., cue dominance, in an opposed cues test. According to the exploration mediation hypothesis, original discrimination acquisition of the peripheral wall cues should be more difficult for the high- than the moderate-drive groups. Furthermore, it was expected that only moderately deprived rats would be able to utilize their initially learned and the incidentally placed redundant-relevant cues. Highly deprived animals were expected to be able to utilize only their originally acquired cue. The design of the experiment also allowed for the investigation of drive level effects on cue dominance.

\section{METHOD}

\section{Subjects}

Thirty-six male albino rats (Wistar strain) approximately 80 days old at time of training and shaping were purchased from Woodlyn Farms, Guelph, Ontario. Two animals failed to run in the experimental sessions and were replaced by substitute rats from the same breeding stock.

\footnotetext{
Apparatus

A two-choice discrimination box, as previously described (Telegdy \& Cohen, 1971), was employed. Two $10 \times 10 \mathrm{~cm}$ stimulus doors led directly into goal chambers. The side walls formed a V-shaped decision chamber. Each radiating wall
} 